\title{
RESEARCH HIGHLIGHT \\ CasX: a new and small CRISPR gene-editing protein
}

\author{
Hui Yang ${ }^{1}$ and Dinshaw J. Patel ${ }^{2,3}$ \\ Cell Research (2019) 29:345-346; https://doi.org/10.1038/s41422-019-0165-4
}

\begin{abstract}
In a recent paper published in Nature, Liu et al. demonstrate the DNA cleavage characteristics of CasX (a newly identified dual-RNA-guided DNA targeting nuclease) and establish its capacity for genome editing in Escherichia coli and human cells. Furthermore, they capture structural snapshots of CasX in different states by cryo-EM and establish a model of RNA assembly, DNA targeting and sequential cleavage.
\end{abstract}

Bacteria and archaea possess CRISPR-Cas-mediated adaptive immune systems to defend against invading foreign nucleic acids. ${ }^{1}$ The targeting and cleavage are achieved by a single crRNAbound Cas protein in Class 2 CRISPR-Cas systems. ${ }^{2,3}$ Class 2 systems can be subdivided into Type II Cas9 and Type V Cas12 that target DNA, as well as Type VI Cas13 that target RNA. Cas9 and Cas12a/ Cpf1 have been well studied in the past and broadly harnessed for gene editing in various cell types and organisms in prokaryotes and eukaryotes. ${ }^{4-6}$ Both Cas9 and Cas 12 systems discriminate self from non-self DNA targets by protospacer adjacent motifs (PAMs) and generate double strand breaks. However, the binding and cleavage of DNA by Cas 9 and Cas 12 are quite different. Cas 9 recognizes a $3^{\prime}-\mathrm{G}$-rich PAM and produces blunt ends cleaved by the RuvC and $\mathrm{HNH}$ domains, whereas Cas 12 recognizes a $5^{\prime}$-T-rich PAM and produces staggered ends cleaved solely by the RuvC domain.

CasX was identified by metagenomic analysis of bacteria from groundwater and characterized as an RNA-guided DNA nuclease. ${ }^{7}$ It recognizes a $5^{\prime}-T T C N$ PAM and is capable of plasmid interference in $E$. coli when presenting sgRNA (covalently linked crRNA-tracrRNA). It shares no similarity to other reported Cas endonucleases except for a RuvC domain located at the $\mathrm{C}$-terminus. The above features of CasX correlate with those of type V Cas12; however, the size of CasX ( 980 aa) is smaller than those of reported Cas12 ( 1200 aa).

In a recent Nature paper, Liu et al. ${ }^{8}$ found that sgRNA-bound Deltaproteobacteria CasX (DpbCasX) contains 20-nt guide segment and recognizes a TTCN PAM element resulting in dsDNA target cleavage with 10-nt staggered ends. It has been reported that Cas12 exhibits PAM-independent non-specific ssDNA trans-cleavage activity when triggered by binding to a crRNA-guidecomplementary ssDNA. ${ }^{7,9}$ Liu et al. found that the trans-ssDNA cleavage activity of CasX is significantly lower than those of Cas12a and Cas12b. They further investigated the potential of CasX for gene editing in E. coli and human cells. They found that DpbCasX has the capability to cleave the target gene in E. coli cells, while its deactivated counterpart is able to bind target genes and reduce gene expression. Moreover, they also show that the related Planctomycetes CasX (PImCasX) has gene editing ability in human HEK293T cells. All these data shed novel insights into the development of CasX as a new gene editing and CRISPRi tool.

The authors further determined a series of cryo-EM structures of CasX in different states to uncover targeting and cleavage mechanisms. The domain composition of CasX-sgRNA-DNA ternary complexes showed some similarity to that of Cas12; however, each structural element adopts distinct folds. They also made efforts at structure determination of apo CasX and CasX-sgRNA and obtained CasX-sgRNA maps at low resolution $(7.5 \AA)$. In combination with mass spectrometry data, they found that sgRNA assembly and DNA loading trigger domain rearrangements.

The authors used two different dsDNAs to generate CasXsgRNA-DNA ternary complexes to investigate the cleavage mechanism. One dsDNA contains non-target DNA strand (30 bp) and the other forms a full R loop (45 bp). Interestingly, the particle sorting of both complexes revealed two states, namely targetstrand DNA cleavage and non-target-strand DNA cleavage, with two thirds of particles adopting the non-target-strand DNA cleavage state. The authors then proposed a sequential model of DNA cleavage, whereby the non-target strand was cleaved first by the RuvC domain followed by cleavage of the target strand. To test this model, they designed a dsDNA containing extended target strand (45 nt) and a shorter non-target strand (20 nt) to mimic an intermediate state after non-target-strand cleavage. As expected, they found that the majority of particles were shifted to the target-strand cleavage state. Specifically, two domains are critical for DNA cleavage. The target-strand loading (TSL) domain adjacent to the target strand DNA is located at the corresponding position of the Nuc domain of Cas12 and involved in DNA strand separation. The non-target-strand binding (NTSB) domain moves towards and interacts with non-target strand DNA upon DNA binding. The NTSB domain is essential for dsDNA cleavage but not trans-ssDNA cleavage, indicating that this domain is involved in DNA unwinding.

Taken together, the contribution by Liu et al. ${ }^{8}$ has uncovered mechanisms of new Cas member CasX for DNA unwinding, cleavage of both DNA strands, and domain movements during transition between two states. Their findings raise the prospect for development of CasX-based gene editing tools. The commonly used Streptococcus pyogenes Cas9 (SpyCas9, 1368 aa) together with sgRNA and promoters has a molecular weight within the size limitation range of a single adeno-associated virus (AAV). To improve delivery efficiency, one solution is the usage of smaller Cas9 orthologs with similar gene editing efficiency, such as Staphylococcus aureus (SauCas9, 1053 aa), Neisseria meningitidis Cas9 (NmeCas9, $1082 \mathrm{aa}$ ), and S. thermophilus 1 (St1Cas9, $1121 \mathrm{aa}$ ),

\footnotetext{
${ }^{1}$ State Key Laboratory of Molecular Biology, CAS Center for Excellence in Molecular Cell Science, Shanghai Institute of Biochemistry and Cell Biology, Chinese Academy of Sciences, University of Chinese Academy of Sciences, Shanghai 200031, China; ${ }^{2}$ Structural Biology Program, Memorial Sloan-Kettering Cancer Center, New York, NY 10065 , USA and ${ }^{3}$ Biology Department, Southern University of Science and Technology, Shenzhen, Guangdong 518055, China

Correspondence: Hui Yang (yanghui@sibcb.ac.cn) or Dinshaw J. Patel (pateld@mskcc.org)
}

Published online: 16 April 2019 
which are relatively smaller and have been successfully harnessed for gene editing in mammalian cells. ${ }^{10}$ However, unlike SpyCas 9 recognition of GG PAM, these Cas9 orthologs recognize relatively longer PAMs (4-5 nt), resulting in a limited targeting scope. ${ }^{10}$ CasX $(<1000 \mathrm{aa})$ is smaller than these Cas9 and typical Cas12, implying its advantage for AAV-mediated delivery and providing more room for other gene editing accessories or tools. More research remains to be done to optimize CasX-based applications ranging from the impact of PAM targeting scope, to off-target effects, DNA specificity, and editing efficiency in different cell types. The current resolution of the two CasX ternary complex states are in the 3.2 and $4.2 \AA$ range. Further improvements in resolution are required for obtaining atomic models that could provide more detailed information and guide engineering efforts at optimization of CasX applications in the future.
ADDITIONAL INFORMATION

Competing interests: The authors declare no competing interests.

\section{REFERENCES}

1. Marraffini, L. A. Nature 526, 55-61 (2015).

2. Koonin, E. V., Makarova, K. S. \& Zhang, F. Curr. Opin. Microbiol. 37, 67-78 (2017).

3. Makarova, K. S. et al. Nat. Rev. Microbiol. 13, 722-736 (2015).

4. Hsu, P. D., Lander, E. S. \& Zhang, F. Cell 157, 1262-1278 (2014).

5. Wright, A. V., Nunez, J. K. \& Doudna, J. A. Cell 164, 29-44 (2016).

6. Jiang, W. \& Marraffini, L. A. Annu. Rev. Microbiol. 69, 209-228 (2015).

7. Burstein, D. et al. Nature 542, 237-241 (2017).

8. Liu, J. J. et al. Nature 566, 218-223 (2019).

9. Swarts, D. C. \& Jinek, M. Mol. Cell 73, 589-600.e4 (2019).

10. Komor, A. C., Badran, A. H. \& David, R. L. Cell 168, 20-36 (2017). 